\section{Ironing out mucosal healing}

Dendritic cell (DC)-derived hepcidin is required for mucosal healing in the mouse intestine after experimental damage, according to new research. Moreover, hepcidin production by DCs was induced by microbial stimulation and promoted local iron sequestration from the microbiota to facilitate intestinal repair.

Gastrointestinal bleeding and altered iron distribution are common in a range of gastrointestinal diseases as a result of infection, inflammation and cancer. "Our focus was on hepcidin, a well-defined master regulator of systemic iron homeostasis that is abundantly produced by the liver, but its role in intestinal damage and inflammation was previously unknown," explain authors Carole Peyssonnaux and Gregory Sonnenberg.

Mice lacking hepcidin exhibited impaired mucosal healing in the dextran sodium sulfate (DSS)-induced experimental model of intestinal damage and inflammation. Hepcidin was essential for tissue repair but, surprisingly, this process occurred independently of the liver and systemic iron regulation. Crucially, conventional DCs local to the intestine were the dominant source of hepcidin in the DSS mouse model, and bacteria and their products were potent inducers of hepcidin expression in DCs. Importantly, increased hepcidin expression was also observed in intestinal biopsy samples from patients with ulcerative colitis and Crohn's disease versus healthy controls, and conventional DCs were confirmed to be a major producer of hepcidin in the inflamed intestine of patients with inflammatory bowel disease.

Finally, DC-derived hepcidin acted on ferroportin-expressing phagocytes (macrophages and/or neutrophils) to facilitate mucosal healing in experimental models. DC-produced hepcidin also promoted local iron sequestration from the gut microbiota. This change in local iron bioavailability shaped the gut microbiota, shifting its composition and promoting

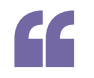

Crucially, DCs local to the intestine were the dominant source of hepcidin g

\title{
PDAC resistance to immunotherapy - a role for autophagy?
}

Pancreatic ductal adenocarcinoma (PDAC) is resistant to immunotherapies, but the mechanisms underlying this resistance are largely unknown. Now, a new study shows that in PDAC tumour cells, major histocompatibility complex class I (MHC-I) molecules are targeted for degradation by an autophagy-dependent mechanism and that inhibiting autophagy can enhance the antitumour response to immune checkpoint blockade (ICB).

First, in human PDAC cell lines, the researchers established that MHC-I molecules, which are important for immune activation, are predominantly localized in autophagosomes and lysosomes rather than at the cell surface. Genetic inhibition of essential autophagy genes was sufficient to restore MHC-I on the surface of PDAC cells. In co-culture experiments with mouse PDAC cells expressing a mutant autophagy protein (ATG4B), increased surface expression

\section{inhibiting} autophagy can enhance the antitumour response to immune checkpoint blockade 5 of MHC-I and antigen presentation was observed, leading to enhanced $\mathrm{CD} 8^{+} \mathrm{T}$ cell-mediated tumour killing. Next, using orthotopic transplantation of PDAC cells into syngeneic mice, autophagy-inhibited cells were found to form smaller tumours, with higher MHC-I expression and increased infiltrating $\mathrm{CD} 8^{+} \mathrm{T}$ cells, than non-autophagy-inhibited cells. "Our study uncovers a new strategy that PDAC cells use to hide from the immune system," says Rushika Perera, co-corresponding author.

Next, the researchers investigated whether autophagy inhibition can influence the response of PDAC cells to ICB. Treatment of syngeneic mice bearing orthotopic mutant ATG4B tumours with antibodies to PD1 and CTLA4 resulted in an enhanced antitumour response in autophagy-inhibited tumours but not in control tumours. Finally, combining
Severe damage, inflammation and impaired mucosal healing in mouse intestine (section staine with haematoxylin and eosin). Image courtesy of G.F. Sonnenberg, Weill Cornell Medicine, USA.

colonization of tissue-protective microorganisms (such as Bifidobacterium spp.) and inhibition of tissue-infiltrating bacteria.

"Our research identifies a new pathway that is important to promote mucosal healing, a major unmet need in inflammatory bowel disease, gastrointestinal infections and colorectal cancers," say Peyssonnaux and Sonnenberg, who plan to test local delivery of hepcidin as a novel therapeutic strategy to promote or boost mucosal healing in experimental mouse models. "Our research also identifies a new pathway controlling local iron distribution in the gut and outlines the ability to employ nutritional immunity for shaping the microbiota," they add.

Katrina Ray

ORIGINAL ARTICLE Bessman, N. J. et al.

Dendritic cell-derived hepcidin sequesters iron from the microbiota to promote mucosal healing. Science 368, 186-189 (2020)

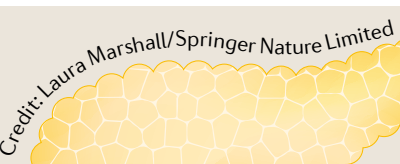

chloroquine, an autophagy inhibitor, with antibodies to PD1 and CTLA4 elicited a potent antitumour response.

The findings suggest that autophagic flux can influence the immunogenicity of PDAC cells and that inhibiting autophagy can sensitize PDAC cells to immunotherapy. "From a clinical perspective, it will be important to understand how best to combine chemotherapy with hydroxychloroquine and immunotherapy in patients to achieve the most effective outcomes," says Perera. "We also want to uncover the underlying reasons as to why MHC-I is targeted for removal by the autophagy-lysosome pathway."

Jordan Hindson

ORIGINAL ARTICLE Yamamoto, K. et al. Autophagy promotes immune evasion of pancreatic cancer by degrading MHC-I. Nature 581, 100-105 (2020) 